\title{
Decreasing nanofluid droplet heating time with alternating magnetic fields
}

\author{
F. F. Fachini ${ }^{1, a)}$ and A. F. Bakuzis $\left.{ }^{2, b}\right)$ \\ ${ }^{1}$ Laboratório de Combustão e Propulsão, Instituto Nacional de Pesquisas Espaciais, \\ 12630-000 Cachoeira Paulista, SP, Brazil \\ ${ }^{2}$ Instituto de Física, Universidade Federal de Goiás, 74001-970 Goiânia, GO, Brazil
}

(Received 17 June 2010; accepted 14 August 2010; published online 22 October 2010)

\begin{abstract}
In this work we propose a new method to decrease the heating time of droplets. Our model considers the heating process of magnetic nanofluid droplet, which was taken to an ambient atmosphere at high temperature and with an alternating magnetic field. Analytical solutions were obtained in systems governed by Brownian and/or low-barrier Néel relaxation (superparamagnetic regime). The droplet heating time was shown to scale with the reciprocal of the square of frequency $\left(1 / f^{2}\right)$ at the low frequency regime. The droplet heating time was calculated as function of frequency for different particle sizes, coating layers, and relaxation mechanisms. (C) 2010 American Institute of Physics.
\end{abstract} [doi:10.1063/1.3489983]

\section{INTRODUCTION}

Nanofluids consist of liquid carriers containing stably dispersed nanoparticles. Those systems have several applications ranging from biomedical, diagnosis, and treatment of diseases, to technological ones. In the later ones enhancing heat transport properties of colloids had received great attention. As for instance, recent experimental results have shown an anomalous thermal conductivity enhancement by introducing very small amounts of copper nanoparticles, carbon nanotubes, magnetite nanoparticles, among other colloidal particles in the liquid carriers. ${ }^{1-7}$ In addition, enhanced ignition properties has been found on fuels containing aluminum nanoparticles. ${ }^{5}$ Besides better heat transfer properties nanoparticles also act as catalysts and can help on the reduction in pollutant production. ${ }^{6}$ Basically, nanofluids are promising systems to enhance the efficiency of ignition and combustion of liquid fuels. In the latter case decreasing the fuel droplets lifetime is crucial. ${ }^{5}$ The general strategy to achieve this goal is to decrease the size of the fuel droplets. Another recent alternative is to introduce small amounts of nanostructures into the fuel, since higher effective liquid thermal conductivity decreases the heating time of the droplets. ${ }^{5}$

In this work we propose another alternative way of decreasing the heating time of droplets through the use of magnetic nanofluids under alternating magnetic fields. The idea is to use the magnetic hyperthermia as another source of heating to enhance the performance of the combustion of fuels. Magnetic nanofluids are more commonly known in the literature as magnetic fluids or ferrofluids. ${ }^{8}$ This system consists of surface-coated magnetic nanoparticles stably dispersed in a liquid carrier (polar or non polar fluids). Since its first use on the mid 1960s, on the control of the flux of fuel under microgravity conditions, several others applications have appeared, as for instance, heat controlled devices through the use of thermomagnetic convection, for example, liquid-cooled loudspeakers ${ }^{9}$ and high power

\footnotetext{
${ }^{a}$ Electronic mail: fachini@lcp.inpe.br.

b)Electronic mail: bakuzis@if.ufg.br.
}

transformers, ${ }^{10,11}$ stem cell labeling, and the diagnosis of diseases, since the nanoparticles are good contrast agents for nuclear magnetic resonance imaging, ${ }^{12}$ or oncological treatment through the phenomena of magnetic hyperthermia. ${ }^{13,14}$

Magnetic hyperthermia consists of the increase in temperature of magnetic fluids due to their response to an alternating magnetic field. Through the use of the first law of thermodynamics, Debye relaxation model and the magnetization equation of motion $\partial M / \partial t=\left(M_{0}-M\right) / t_{m}$, Rosensweig ${ }^{8}$ was able to derive an expression for the nanofluid power dissipation, which was found to be

$$
P=\pi \mu_{0} \xi_{0} H_{0}^{2} \bar{f} \frac{2 \pi f t_{m}}{1+\left(2 \pi f t_{m}\right)^{2}},
$$

with $\mu_{0}$ the permeability of free space $\left(\mu_{0}=4 \pi 10^{-7} \mathrm{~N} / \mathrm{A}^{2}\right)$, $H_{0}$ the amplitude of the alternating applied magnetic field, $f$ the frequency, $\xi_{0}$ is the chord susceptibility, $\xi_{0}=\left(3 \zeta_{i} / \zeta\right)$ (coth $\zeta-1 / \zeta$ ), assumed to be weakly temperature dependent; where $\xi_{i}$ is Langevin initial susceptibility $\left(\xi_{i}\right.$ $=\phi \mu_{0} M_{s}^{2} V / 3 k_{B} T, \phi$ is the particle volume fraction) and $\zeta$ $=\mu_{0} M_{s} H_{0} V / k_{B} T$, with $H=H_{0} \cos (2 \pi \bar{f} t)$ and $M_{s}$ the saturation magnetization of the nanoparticle. $t_{m}$ is the effective relaxation time, which has two contributions, namely the Brownian $\left(t_{B}\right)$ and the Néel relaxation times $\left(t_{N}\right), 1 / t_{m}$ $=1 / t_{B}+1 / t_{N}$. The extrinsic Brownian relaxation time, whose origin is related to the rotation of the nanoparticle with the change in the magnetic field orientation, is given by $t_{B}$ $=3 \eta V_{H} / k_{B} T$ with $\eta$ the liquid viscosity, $k_{B}$ the Boltzmann constant, $T$ the temperature, and $V_{H}$ the hydrodynamic volume of the particle, which consists of the nanoparticle together with the molecule coating layer, $V_{H}=\pi(D+2 \delta)^{3} / 6$, in which $D$ and $\delta$ are the nanoparticle diameter and the coating layer thickness, respectively. On the other hand, the intrinsic Néel relaxation time, following Brown's derivation ${ }^{8,15}$ for the low barrier case, is given by $t_{N}=\tau_{0} \Gamma /(1-2 \Gamma / 5)$, for $\Gamma$ $<1$ in which $\Gamma \equiv K V_{M} / k_{B} T$. The Néel relaxation time is related to the rotation of the magnetic moment of the nanoparticle along the anisotropy axis. On the low barrier limit $(\Gamma$ $\ll 1$, i.e., the superparamagnetic limit) one obtains $t_{N}=\tau_{0} \Gamma$, in 

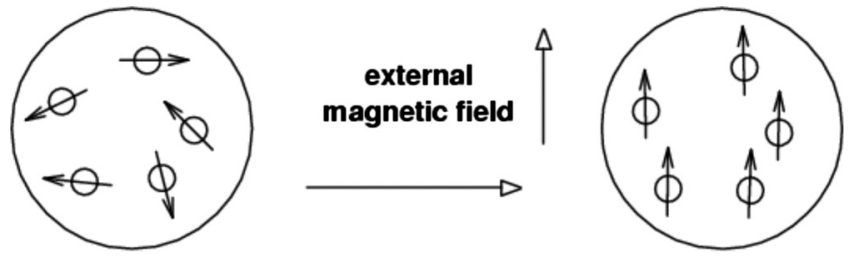

molecular collision with the nanoparticles
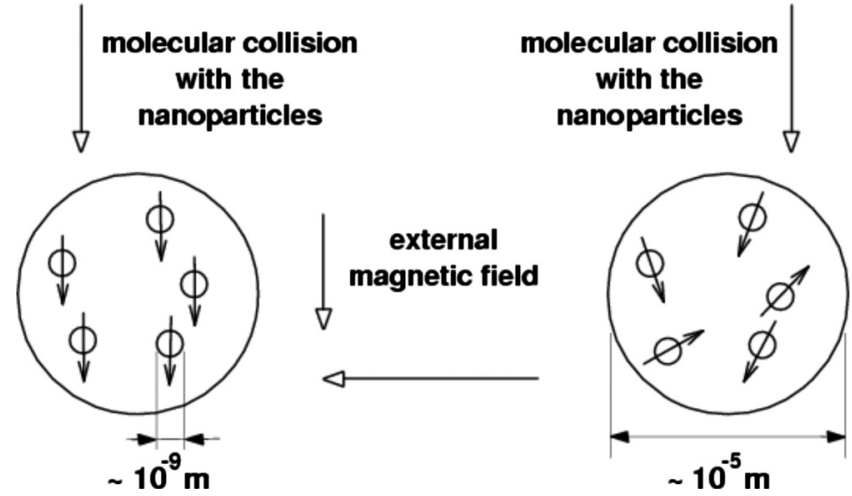

FIG. 1. Schematic representation of a magnetic nanofluid droplet under alternating magnetic field during one cycle.

first approximation. ${ }^{16,17}$ It is important to point out that $V$ is the nanoparticle volume without the coating layer ( $V$ $\left.\equiv \pi D^{3} / 6\right)$, and $K$ is the magnetic anisotropy, which is strongly dependent upon the material, shape and also particle size. ${ }^{18,19}$ The process of heat generation in magnetic nanofluids under the influence of a magnetic field is due to in part by the mobility of the nanoparticles inside the fluid and in part by heat conduction from the nanoparticle to the liquid carrier.

Figure 1 shows a schematic representation of a nanofluid droplet under alternating magnetic field during one cycle. The droplet is represented as a circle with a typical size of $10^{-5} \mathrm{~m}$, which contains magnetic nanoparticles with diameter on the order of $10^{-9} \mathrm{~m}$. The nanoparticles magnetic dipoles try to align with the applied magnetic field. Both mechanisms, Brownian and Néel relaxation, can be involved on the magnetic moment rotation. On the other hand, molecular collisions with the nanoparticles work against dipole alignment promoting random nanoparticles magnetic moment orientations. During this process heat is generated.

The Rosensweig model predicts a strong dependence of the power dissipated on the nanoparticle composition (ferrite type), mainly due to different magnetic anisotropy constant values, viscosity, and nanoparticle size. In some cases the heating mechanism can be associated to the Néel relaxation, while in others Brownian mechanism dominates. In fact some recent experimental results have shown some agreement with the model. ${ }^{20,21}$ Although, the experimental parameter that is compared with theoretical model just considers the initial slope of the time dependent nanofluid temperature profile, under alternating magnetic field conditions. Indeed, as far as we know, the whole temperature profile time dependence has not been explored analytically until now.

\section{THEORETICAL MODEL}

The problem that we are interested consists of the heating of magnetic nanofluid droplets under an alternating mag- netic field. So we consider that the droplet has a characteristic length $\bar{a}_{0}$ (radius) and temperature $T_{0}$ at the initial condition $(t=0)$. Also, the fluid parameters are the boiling temperature $T_{b}$, density $\rho_{l}$, specific heat $c_{l}$, and thermal conductivity $k_{l}$. The magnetic fluid is assumed to be in ambient atmosphere with density $\rho_{\infty}$ and temperature $T_{\infty}$ (the conditions are such that the perfect gas relation holds $P_{\infty}$ $=R \rho_{\infty} T_{\infty}$ ), the gas phase properties are specific heat at constant pressure $c_{p}$ and thermal conductivity $k_{g}$, which are assumed to have constant values. In addition, the nanofluid has low volatility, i.e., the vaporization only begins when the temperature is very close to the boiling temperature (the vaporization rate $\dot{m}$ becomes important for $T \sim T_{b}$ ). Under these conditions, the heating process occurs practically with no change in the droplet dimensions.

In order to handle properly the problem, the liquid phase energy conservation equation is normalized using the following definitions $\tau \equiv t / t_{c}, x \equiv r / \bar{a}_{0}, \quad \theta \equiv T / T_{b}$. The time $t_{c}$ $\equiv\left[a_{0}^{2} /\left(k_{g} / c_{p} \rho_{\infty}\right)\right]\left(\rho_{l} / \rho_{\infty}\right)$ is an estimative of the heating time if only the heat conduction from the gas phase is responsible for the heating process, ${ }^{22,23}$ while the initial temperature is $\theta_{0}$. So the dimensionless energy conservation of the liquid phase can be written as ${ }^{24}$

$$
\frac{\partial \theta}{\partial \tau}=\frac{A}{x^{2}} \frac{\partial}{\partial x}\left(x^{2} \frac{\partial \theta}{\partial x}\right)+P_{m} \frac{f^{2} \tau_{m}}{1+\left(f \tau_{m}\right)^{2}},
$$

in which $A \equiv c_{p} k_{l} / c_{l} k_{g}$, the dimensionless frequency $f$ $=2 \pi \bar{f} t_{m}^{*}\left(t_{m}^{*}\right.$ defined below $)$ and the dimensionless power dissipation term corresponds to

$$
P_{m}=\frac{\mu_{0} \chi_{0} H_{0}^{2} / 2}{\rho_{l} c_{l} T_{b}} \frac{t_{c}}{t_{m}^{*}} .
$$

The dimensionless relaxation time $\tau_{m} \equiv t_{m} / t_{m}^{*}$ is given by ${ }^{8,16}$

$$
\begin{aligned}
\tau_{m} \equiv & \frac{(1+\Delta)\left(1-2 \Gamma^{*} / 5\right) /\left[\Delta\left(1-2 \Gamma^{*} / 5\right)+1\right]}{\theta-\left(2 \Gamma^{*} / 5\right) /\left[\Delta\left(1-2 \Gamma^{*} / 5\right)+1\right]}, \\
& \text { for } \quad \Gamma^{*} / \theta<1
\end{aligned}
$$

The effective relaxation time $t_{m}$, defined for the temperature $T$, is $t_{m} \equiv t_{N} t_{B} /\left(t_{B}+t_{N}\right)$, with $t_{m}^{*}$ the effective relaxation time determined at the boiling temperature $\theta=1\left(T=T_{b}\right)$. The Brownian relaxation time $t_{B}$, defined as $t_{B} \equiv t_{B}^{*} / \theta$ where $t_{B}^{*}$ $=3 \eta V_{H} / k_{B} T_{b}$, and the Néel relaxation time $t_{N}$, given by $t_{N}$ $\equiv t_{N}^{*}\left(1-2 \Gamma^{*} / 5\right) /\left(\theta-2 \Gamma^{*} / 5\right)$, for $\Gamma^{*} / \theta<1$ were used in the derivation of Eq. (3). The definition of $t_{N}^{*}$ is $t_{N}^{*}$ $\equiv \tau_{0} \Gamma^{*} /\left(1-2 \Gamma^{*} / 5\right)$ for $\Gamma^{*} / \theta<1$ with $\Gamma^{*}$ equals to $K V / k_{B} T_{b}$. The parameter $\Delta$ measures the ratio of the Néel relaxation time $t_{N}^{*}$ to the Brownian relaxation time $t_{B}^{*}\left(\Delta \equiv t_{N}^{*} / t_{B}^{*}\right)$ and is expressed by $\Delta \equiv\left(\tau_{0} / t_{B}^{*}\right) /\left(1 / \Gamma^{*}-2 / 5\right)$.

For the superparamagnetic case $\Gamma^{*} \ll 1$, the Néel relaxation time is expressed by $t_{N} \equiv \tau_{0} \Gamma^{*} / \theta .^{17}$ The expression for the effective relaxation time $\tau_{m, s p}$, considering both mechanisms Néel and Brownian, in the superparamagnetic regime is found by imposing $\Gamma^{*} \ll 1$ into Eq. (3), $\tau_{m, s p} \equiv t_{m, s p} / t_{m, s p}^{*}$ $=1 / \theta$, in which the effective relaxation time at boiling temperature $(\theta=1) t_{m, s p}^{*}$ is given by $t_{m, s p}^{*} \equiv \tau_{0} /\left(1 / \Gamma^{*}+\tau_{0} / t_{B}^{*}\right)$. It is interesting to notice that the $\theta$ dependence is the same as 
considering only the Brownian relaxation process. Finally, the nondimensional boundary and initial conditions are

$$
\begin{aligned}
\left.\frac{\partial \theta}{\partial x}\right|_{x=0} & =0,\left.\quad x^{2} \frac{\partial \theta}{\partial x}\right|_{a^{+}}-\left.\frac{k_{l}}{k_{g}} x^{2} \frac{\partial \theta}{\partial x}\right|_{a^{-}}=\lambda l, \quad \theta \\
& =\theta_{0}, \quad \text { at } \quad \tau=0, \quad x<a,
\end{aligned}
$$

where nondimensional vaporization rate $\lambda$ is $\dot{m} c_{p} /\left(4 \pi \bar{a}_{0} k_{g}\right)$, the latent heat $l$ is $L /\left(c_{p} T_{b}\right)$ and nondimensional droplet radius is $a \equiv \bar{a} / \bar{a}_{0}$.

In this work, the hypothesis of very low volatile liquid is considered, therefore the magnetic fluid heating occurs practically with negligible vaporization $(\lambda \ll 1)$, i.e., no volume change $(a=1)$. Also, the magnetic power is considered much larger than the thermal conduction from the gas phase, so $P_{m} \gg 1$. Therefore, the Laplacian term can be neglected under this condition. The solution of the dimensionless energy conservation equation, in the high magnetic power condition, considering only the Brownian relaxation time, is

$$
P_{m, B} \tau=\frac{\theta^{2}-\theta_{0}^{2}}{2 f^{2}}+\ln \left(\frac{\theta}{\theta_{0}}\right),
$$

with $f \equiv 2 \pi \bar{f} t_{B}^{*}$ and $t_{m}^{*}=t_{B}^{*}$. At the high frequency limit $(f$ $\gg 1)$ the temperature evolution is $\theta=\theta_{0} \exp \left(P_{m, B} \tau\right)$. Under this condition, the appropriate time scale is $\tau \sim\left(P_{m, B}\right)^{-1}$. On the contrary, for $f \ll 1$, low frequency regime, the solution (5) simplifies to $\theta=\left(\theta_{0}^{2}+2 f^{2} P_{m, B} \tau\right)^{1 / 2}$ and the appropriate time scale $\tau \sim\left(f^{2} P_{m, B}\right)^{-1}$ depends on the frequency. On the other hand, considering only the low-barrier Néel case, also known as the superparamagnetic one, the relaxation time $\tau_{m, s p}$ for $\Gamma^{*} \ll 1$ has the same dependence on the temperature $\theta$ as Brownian relaxation time. As a consequence the solution for Eq. (2) is the same as Eq. (5), unless by a factor multiplying the Brownian magnetic power parameter $P_{m, B}$,

$$
P_{m, s p} \tau=\left(P_{m, B} / \Delta\right) \tau=\frac{\theta^{2}-\theta_{0}^{2}}{2 f^{2}}+\ln \left(\frac{\theta}{\theta_{0}}\right),
$$

and that $f \equiv 2 \pi \bar{f} t_{N}^{*}$, and $t_{m}^{*}=t_{N}^{*}$, instead. Note that so far we had obtained the solution of both isolated relaxation mechanisms. In addition, the adopted form of presenting the problem allowed us to write the heating time $P_{m, i} \tau_{h}$ as a function of few parameters: dimensionless frequency $f$ and the ratio of the magnetic power to the thermal power $P_{m}$, both dependent upon the relaxation mechanism. Also, in the present work, the condition $P_{m} \gg 1$ is analyzed. It is important to point out that this condition implies an uniform heating, except in a very narrow zone close to the droplet surface, which will be studied in a future analysis.

\section{RESULTS AND DISCUSSION}

We obtained the analytical solutions of the droplet heating time for several specific cases. Now, we are in position to analyze those results. Let's start considering only the viscous dissipation heat generation, i.e., the case where heating is governed only by the Brownian relaxation time. In this case the ferrofluid heating time $P_{m, B} \tau_{h}$ as function of the dimensionless frequency $f$ is specified by the Eq. (5) with the condition $\theta=1$. Figure 2(a) shows the heating time $P_{m, B} \tau_{h}$ as function of the dimensionless frequency for different initial temperature conditions $\theta_{0}=0.75,0.85,0.95$. Note the strong influence upon the heating time at the low frequency regime, while at the high frequency range no frequency dependence is obtained. In fact, for low frequencies, the heating time $P_{m, B} \tau_{h}$ varies as $f^{-2}$, and for high frequencies, $P_{m, B} \tau_{h}$ reaches a constant value. The influence of the initial temperature $\theta_{0}$ is also exposured on Fig. 2(a), which shows that increasing $\theta_{0}$ the heating time decreases as expected.

On the other hand, the Néel relaxation time for the lowbarrier case, also known as superparamagnetic condition $\left(\Gamma^{*} \rightarrow 0\right)$, has the same temperature dependence as the Brownian relaxation time [see Eq. (6)]. Therefore the heating time $P_{m, s p} \tau_{h}$ varies similarly as $P_{m, B} \tau_{h}$ with the frequency. As a consequence one can use the same figure [Fig. 2(a)] to represent this situation. The only important thing that should be noticed is that the dimensionless power dissipation $P_{m, s p}$ in this case is different being related to $P_{m, B}$ according to $P_{m, s p}=\left(t_{B}^{*} / t_{m, s p}^{*}\right) P_{m, B}=P_{m, B} / \Delta$.

Although, dimensionless parameters are excellent for theoretical analysis, sometimes it is useful to obtain results considering typical experimental values from the literature. As for example, on magnetic hyperthermia experiments, usually, the frequency range investigated is below $1 \mathrm{MHz}$. Therefore, we decided to investigate the heating time frequency dependence using those typical ranges. In our simulation we considered a typical maghemite nanoparticle with a magnetic anisotropy of $4.6 \mathrm{~kJ} / \mathrm{m}^{3}$ dispersed in a carrier fluid with a viscosity value of $0.00235 \mathrm{~kg} / \mathrm{ms}^{8}{ }^{8}$ Figure 2(b) shows the Brownian heating time $P_{m, B} \tau_{h}$ as function of the frequency (hertz) for different particle sizes (3, 5, 7, and $9 \mathrm{~nm}$ ). In the calculation we considered an initial temperature condition of $\theta_{0}=0.75$, a fluid boiling temperature of $500 \mathrm{~K}$ and a coating layer thickness of $2 \mathrm{~nm}$. At the low frequency range, we found that the larger the particle size the lower is the normalized heating time. However, for frequencies close to 1 $\mathrm{MHz}$ the heating time approaches a constant value.

An important discussion about the hyperthermia phenomena is which relaxation process can increase the temperature more efficiently. In order to get into a conclusion about this point we compared both isolated relaxation mechanisms. In Fig. 2(c) we show the ratio of the Brownian heating time to the low barrier Néel one as function of the frequency for different particle sizes. Interesting at a very broad frequency range (lower frequencies) the ratio is almost a constant, while at higher frequency values it start to increase. Also, the ratio was shown to be smaller for lower particle sizes. More important the theoretical results clearly show that isolated Brownian heating time mechanism is more efficient than the Low Barrier Néel one. In addition, in Fig. 2(d) we show the ratio of the Brownian heating time to the Low Barrier Néel one as function of the frequency for different coating layers thickness. Calculations were performed for a particle diameter of $7 \mathrm{~nm}$. At lower frequencies we found that the larger the coating layer the lower is the ratio value, while at higher frequencies the opposite was observed (Fig. 2).

Note that, so far, we had only compared both isolated relaxation mechanisms, from which we get into the conclu- 

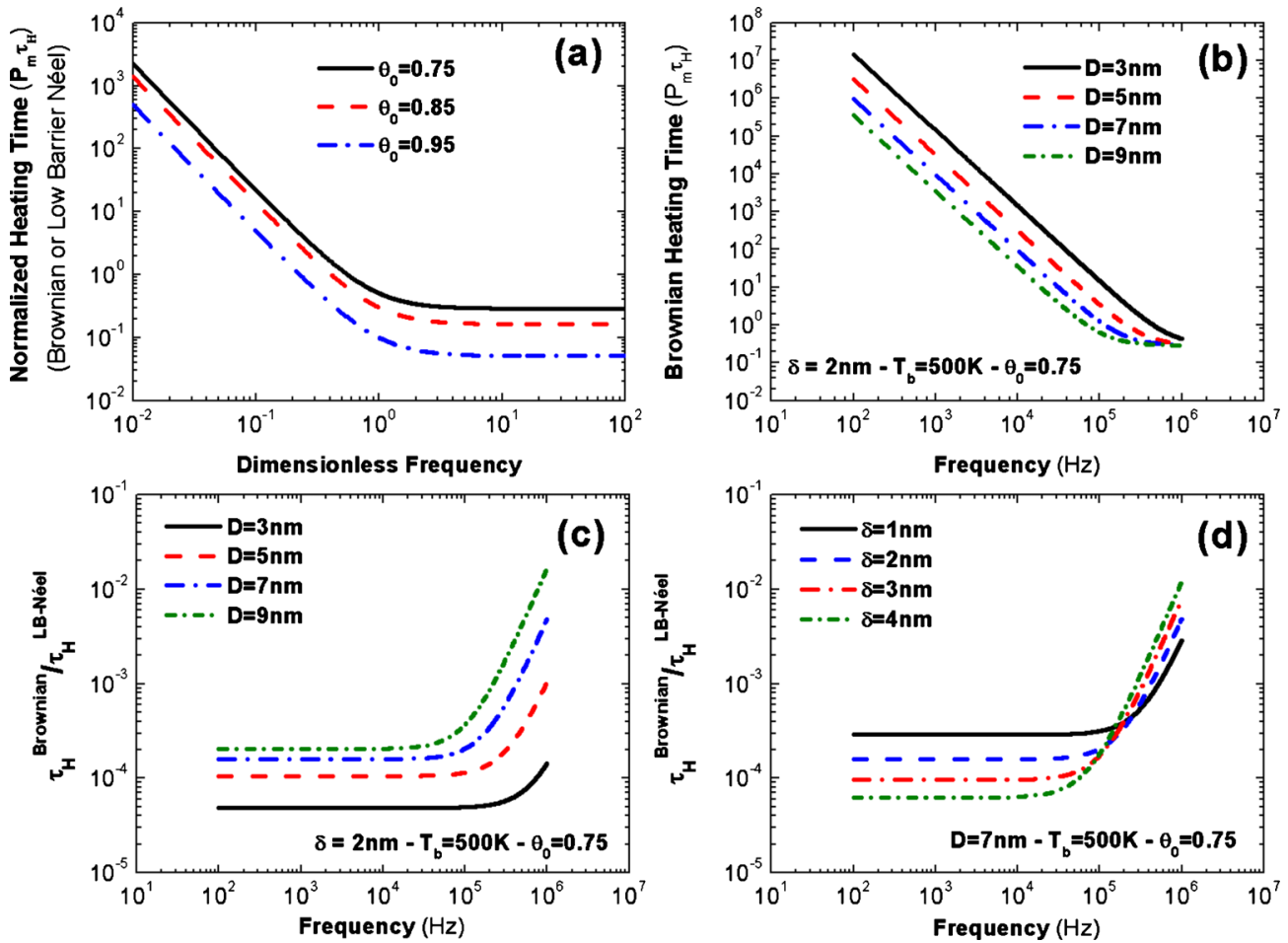

FIG. 2. (Color online) (a) The heating time $P_{m, B} \tau_{h}$ considering only the Brownian relaxation mechanism or the low barrier Néel case as a function of the dimensionless frequency $f$ for three initial temperatures $\theta_{0}=0.75,0.85,0.95$. (b) Brownian heating time as function of frequency for different particle sizes. (c) Ratio of the Brownian heating time to the low barrier Néel one as function of frequency for different particle sizes. (d) Ratio of the Brownian heating time to the low barrier Néel one as function of frequency for a nanoparticle diameter of $7 \mathrm{~nm}$ and different coating layer sizes.

sion that Brownian relaxation is more efficient on heating magnetic nanofluids at a frequency range below $1 \mathrm{MHz}$. Although the result is correct in reality one always have both mechanisms competing. Following, Shliomis ${ }^{25}$ the effective relaxation time is $1 / t_{m}=1 / t_{B}+1 / t_{N}$. It is not difficult to show that at the boiling temperature $t_{m}^{*}=t_{N}^{*} /(1+\Delta)$. In the maghemite-based nanosystems analyzed in this work $(\Delta$ $\ll 1)$. Therefore the active relaxation mechanism is in fact the low barrier Néel one. Indeed, a similar conclusion was obtained before by Fortin et al. ${ }^{20}$ So, unfortunately, the heating process occurs through the less effective mechanism for this application. Indeed, the solution of the dimensionless energy conservation equation, considering both mechanisms, in the superparamagnetic regime $\left(\Gamma^{*} \ll 1\right)$, is given by

$$
P_{m, e f} \tau=(1+\Delta) P_{m, s p} \tau=\frac{\theta^{2}-\theta_{0}^{2}}{2 f^{2}}+\ln \left(\frac{\theta}{\theta_{0}}\right),
$$

with the dimensionless frequency $f \equiv 2 \pi \bar{f} t_{m}^{*}$. Therefore, we conclude that the droplet heating time has the same frequency dependence for three cases: isolated Brownian relaxation mechanism, isolated low-barrier Néel one and finally the situation considering both cases together, i.e., Brownian together with low-barrier Néel relaxation mechanism.

After identifying the heating mechanism of maghemitebased nanoparticles, it is important to investigate its diameter dependence. Figure 3 shows the ratio of the effective heating time of a nanoparticle of size $D$ to the lowest one investigated $(3 \mathrm{~nm})$. In this calculation, we considered the same particle volume fraction and the Langevin susceptibility. We found that the larger the particle size the more efficient is the heating mechanism. Note, that only the ratio is frequency independent. In fact, one, obviously, still obtain a heating time scaling with $f^{-2}$ at low frequencies, while at the high frequency range it approaches a constant value. Another important issue is that for larger particle sizes the low barrier Néel expression is not valid anymore. In this case one should use the high barrier Néel equation, ${ }^{16,17}$ which will result on another theoretical solution for the energy conservation equation. Such investigation will be performed in the near future. Nevertheless, the results obtained for the superparamagnetic regime (Fig. 2) of maghemite-based nanofluids, at this particle size range, are in agreement with theoretical and experimental results from the literature..$^{8,20,21}$ One should keep in mind that, different from previous works, our results corresponds to an analysis of the whole time dependence temperature profile.

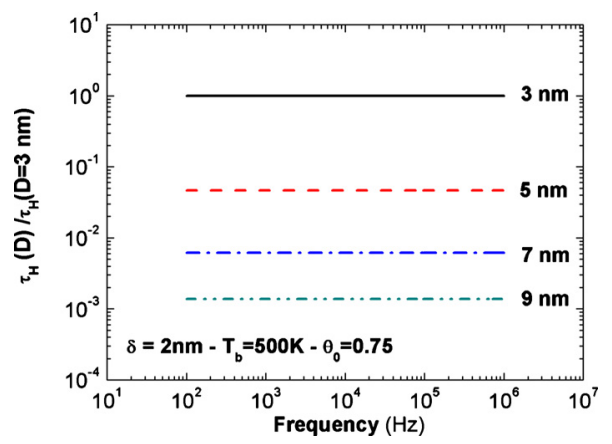

FIG. 3. (Color online) Ratio of the effective heating time of a nanoparticle of diameter $D$ to the lowest investigated $(3 \mathrm{~nm})$ is a function of the frequency (hertz). The theoretical calculations considered the initial droplet temperature of $\theta_{0}=0.75$, a boiling temperature of $500 \mathrm{~K}$, a coating layer thickness of $2 \mathrm{~nm}$ and particle sizes of 3,5,7, and $9 \mathrm{~nm}$. 


\section{CONCLUSIONS}

In conclusion, we showed that the droplet heating time of magnetic nanofluids can be decreased with alternating magnetic fields. A possible application of this phenomenon is to the combustion of liquid fuels, for example, diesel. There is, of course, a challenge associated to the viability of applying such alternating magnetic fields, particularly, high frequency ones. Nevertheless, we found interesting effects already at the low frequency range. Since there are still some environmental and efficiency issues, particular with diesel fuels, such application might be interesting. On the other hand, the theoretical calculations shown in this paper, might have other applications, as for instance, on the biomedical ground, where alternating magnetic fields are being used to oncological treatment. Indeed, our analytical solutions for the energy conservation equation, within the whole temperature profile time dependence, might be extremely useful on developing nanostructures for hyperthermia applications.

F.F.F. acknowledges financial support from CNPq under the Grant Nos. 563835/2008-7 and 552417/2009-2 and in part by CAPES under the Grant PNPD. F.F.F. also thanks Dr. Antonio C. Seridonio for helpful discussions. A.F.B. thanks financial support from CNPq, CAPES, FINEP, and FUNAPE.

${ }^{1}$ J. A. Eastman, S. U. S. Choi, S. Li, W. Yu, and L. J. Thompson, Appl. Phys. Lett. 78, 718 (2001).

${ }^{2}$ S. U. S. Choi, Z. G. Zhang, W. Yu, F. E. Lockwood, and E. A. Grulke, Appl. Phys. Lett. 79, 2252 (2001).
${ }^{3}$ P. Keblinski, R. Prasher, and J. Eapen, J. Nanopart. Res. 10, 1089 (2008). ${ }^{4}$ P. D. Shima, J. Philip, and B. Raj, Appl. Phys. Lett. 94, 223101 (2009).

${ }^{5}$ H. Tyagi, P. E. Phelan, R. Prasher, R. Peck, T. Lee, J. R. Pacheco, and P. Arentzen, Nano Lett. 8, 1410 (2008).

${ }^{6}$ R. A. Yetter, G. A. Rishs, and S. F. Son, Proc. Combust. Inst. 32, 1819 (2009).

${ }^{7}$ J. Philip, P. D. Shima, and B. Raj, Appl. Phys. Lett. 91, 203108 (2007).

${ }^{8}$ R. E. Rosensweig, J. Magn. Magn. Mater. 252, 370 (2002).

${ }^{9}$ K. Raj and R. Moskowitz, J. Magn. Magn. Mater. 85, 233 (1990).

${ }^{10}$ V. Segal, A. Rabinovich, D. Nattrass, K. Raj, and A. Nunes, J. Magn. Magn. Mater. 215-216, 513 (2000).

${ }^{11}$ F. R. Cunha, H. L. G. Couto, and N. B. Marcelino, Magnetohydrodynamics 43, 421 (2007).

${ }^{12}$ J. W. M. Bulte, T. Douglas, B. Witwer, S. C. Zhang, E. Strable, B. K. Lewis, H. Zywicke, B. M. B, P. van Gelderen, P. B. M. Moskowitz, I. D. Duncan, J. A. Frank, Nat. Biotechnol. 19, 1141 (2001).

${ }^{13}$ A. Jordan, R. Scholz, P. Wust, F. Horst, and F. Roland, J. Magn. Magn. Mater. 201, 413 (1999).

${ }^{14}$ C. L. Dennis, A. J. Jackson, J. A. Borchers, P. J. Hoopes, R. Strawbridge, A. R. Foreman, J. van Lierop, C. Gruttner, and R. Ivkov, Nanotechnology 20, 395103 (2009).

${ }^{15}$ J. W. F. Brown, Phys. Rev. 130, 1677 (1963).

${ }^{16}$ H.-D. Pfannes, A. Mijovilovich, R. Magalhães Paniago, and R. Paniago, Phys. Rev. B 62, 3372 (2000).

${ }^{17}$ P. C. Fannin and S. W. Charles, J. Phys. D: Appl. Phys. 27, 185 (1994).

${ }^{18}$ F. Bødker, S. Mørup, and S. Linderoth, Phys. Rev. Lett. 72, 282 (1994).

${ }^{19}$ A. F. Bakuzis, P. C. Morais, and F. Pelegrini, J. Appl. Phys. 85, 7480 (1999).

${ }^{20}$ J. P. Fortin, C. Wilhelm, J. Servais, C. Menager, J. C. Bacri, and F. Gazeau, J. Am. Chem. Soc. 129, 2628 (2007).

${ }^{21}$ M. Lévy, C. Wilhelm, J. M. Siaugue, O. Horner, J. C. Bacri, and F. Gazeau, J. Phys.: Condens. Matter 20, 204133 (2008).

${ }^{22}$ F. F. Fachini, Combust. Flame 116, 302 (1999).

${ }^{23}$ F. F. Fachini, A. Liñáan, and F. A. Williams, AIAA J. 37, 1426 (1999).

${ }^{24}$ S. Maenosono and S. Saita, IEEE Trans. Magn. 42, 1638 (2006).

${ }^{25}$ M. I. Shliomis, Sov. Phys. Usp. 17, 153 (1974). 